\title{
CCD PARALLAXES FOR FAINT SOUTHERN HIGH PROPER MOTION STARS
}

\author{
C.A. ANGUITA, M.T. RUIZ \\ Obs. Astronomico Nacional \\ Universidad de Chile \\ Casilla 36-D \\ Santiago, Chile
}

\begin{abstract}
In April 1985, we started a program to measure trigonometric parallaxes for faint southern high proper motion stars, using a CCD at the Cassegrain focus of the Cerro Tololo Interamerican Observatory (CTIO) 1.5 -m telescope. The program stars $\left(m_{R}>16 ; \mu \geq 1\right.$ arcsec/year) were selected from the LHS Catalogue and the University of Chile proper motion program.

The $\mathrm{X}$ and $\mathrm{Y}$ positions of the stellar image centroids were obtained using the algorithms of DAOPHOT program packages. The precision of the measurement of one stellar image is about 6 milliarcseconds $(0.02$ pix $)$. For stars with $16<\mathrm{m}_{\mathrm{R}}<19.5$ a precision of 2 milliarcseconds in the parallax determination can be obtained in a one year period. Trigonometric parallaxes for some stars common to other parallax programs are given, showing an agreement with those results within the quoted mean errors.
\end{abstract}

Several technical aspects of the present program are also discussed.

\section{Discussion}

van Altena: Is there a difference between the short term repeatability and the long term precision of the CCD observations?

ANGUITA: No, we have found no significant differences in short term and long term precision of our observations. I can say this, since we have studied our CCD results very carefully in relation to these "could-be" differences because during the four years of this ongoing project we have used three different CCD chips from RCA.

SHAKHT: It is known that the proper motions of VB8 and VB10 might have perturbations due to suspected invisible companions. Could you say something about these perturbations?

Angurta: The time-bases of our VB8 and VB10 CCD observations are not large enough to say anything reliable concerning perturbations due to invisible companions. In the futuresome years from now-certainly we will be able to say if there are or are not such perturbations. If I recall correctly, the perturbation period is about 5 years for VB10 and a little longer for VB8.

CHEREPASHCHuK: What value of seeing do you have during your observations?

AngurTa: Better than 1.6 arcsec at the $50 \%$ intensity level. 